\title{
STUDY OF SHORT-RANGE MOTION $\circlearrowleft$ F ATOMIC HYDROGEN IN AMORPHOUS SILICON BY NEUTRON REFLECTOMETRY
}

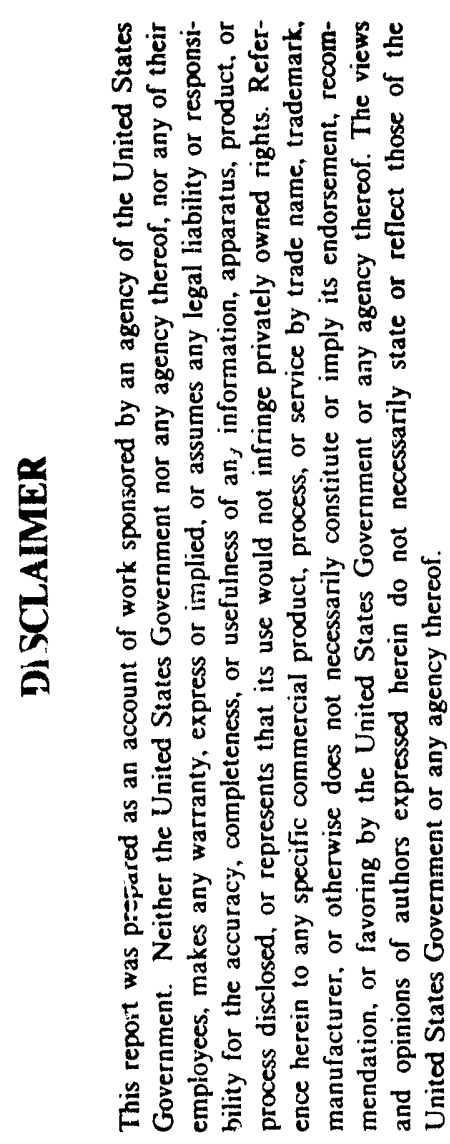

\author{
W. D. Dozier \\ Intense Pulsed Neutron Division \\ Argonne National Laboratory \\ Argonne, IL 60439 \\ K. W. Herwig \\ University of Missouri Research Reactor Facility \\ Columbia, MO 65211 \\ R. Shinar \\ Microelectronics Research Center \\ Iowa State University \\ Ames, IA 50011 \\ H. Jia and J. Shinar \\ Ames Laboratory-USDOE and \\ Physics and Astronomy Department \\ Iowa State University \\ Ames, IA 5001i

\begin{abstract}
The submitted manuscipt has been authored by a
contractor of the U.S. Government under contrad No.

W.31-109.ENG.38. Aconidingly, the U.S. Government

relains a nonexclusive, ruyahy-tree license to publish

or resroduce the published form of this contibution, of
\end{abstract} \\ allow others to do so, for U.S. Government purposes. \\ May, 1992
}

JUN 241992

/sm

SUBMITTED TO: Proceedings of 1992 Spring Meeting of the MRS, San Francisco, CA

This work is supported by the U.S. Department of Energy, Basic Energy Sciences, Materials

Sciences, under contract No. W-31-109-ENG-38. 


\title{
STUDY OF SHORT-RANGE MOTION OF ATOMIC HYDROGEN IN AMORPHOUS SILICON BY NEUTRON REFLECTOMETRY
}

\author{
W. D. Dozier, ${ }^{*}$ K. W. Herwig, ${ }^{* *}$ R. Shinar, † H. Jiał and J. Shinart \\ *Argonne National Laboratory, Argonne, IL 60439 \\ **University of Missouri Research Reactor Facility, Columbia, MO 65211 \\ † Microelectronics Research Center, Iowa State University, Ames, IA 50011 \\ $¥$ Ames Laboratory-USDOE and Physics and Astronomy Department, Iowa State University, \\ Ames, IA 50011
}

\begin{abstract}
Preliminary results of neutron reflectometry (NR) measurements on rf sputter-deposited a-Si:H/a-Si:D bilayers indicate that this technique may be used to monitor $H$ and $D$ meions over distances of $\approx 10$ to $200 \AA$ with a nominal resolution of $5-10 \AA$. In studying rf sputterdeposited thin films containing a high density of microvoids annealed at $270 \mathrm{C}$, we found that the hydrogen diffused a distance of only $\approx 100 \AA$. Further annealing at 270 and $280 \mathrm{C}$ produced no additional motion. This result is consistent with a model of this system in which the hydrogen is trapped in microvoids after moving a relatively short distance.
\end{abstract}

\section{Introduction}

Long-range $(\approx 200$ to $3000 \AA)$ diffusion of atomic $\mathrm{H}$ in a-Si:H has been extensively studied by thermal desorption spectroscopy (TDS) and secondary ion mass spectromety (SIMS).[1-12] The strongest motivation for these studies is the mounting evidence for the role of $\mathrm{H}$ motion in the dynamics of carrier recombination-induced defect generation and annealing processes, i.e., the Stäbler-Wronski effect (SWE).[4, 10, 13] The SWE is clearly the most prominent current issue in both the physics and technology of a-Si:H. The detailed nature of the local $\mathrm{H}$ motion, and thus its role in the SWE, remains undecided. Higher-resolution techniques that can directly probe this local ruotion are therefore needed. This paper describes the application of such a technique, neutron reflectometry (nominal resolution $\approx 5-10 \AA$ ), to this problem. Not only does NR have high spatial resolution, but it is extremely sensitive to the $\mathrm{H}$ content of the samples; this provides an additional check on the usual IR measurements. Our preliminary NR results on rf sputter-deposited a-Si:H containing large microvsid fractions show suppression of long-range motion, consistent with previous SIMS results. The NR is also consistent with IR measurements of the $\mathrm{H}$ content of films deposited under the same conditions.

\section{Experimental Procedure}

Samples were typically prepare ${ }^{\prime}$ by ri sputter-deposition of a-Si:D or a-Si:H, on a 2 " diameter, 3/16" thick Si wafer. Nomina' thicknesses were obtained by previous calibrations of the sputtering rate as a function of process parameters, as well as with a profilometer. The samples were deposited on a nominally unheated substrate; the temperature of the growing film, which was heated by electron and ion bombardment, was estimated to be $\approx 150$ C. [14] The of the top iaver has been determined for Samples deposited under similar conditions as HJ13 and HJ34 have shown a total Si-bonded H content of $\approx 20$ atomic \%.[14,15] A summary of the samples discussed in this article is found in Table 1. 


\begin{tabular}{|c|c|c|c|}
\hline Sample ID & Top Layer & Base layer & Rf Power \\
\hline HJ34 & $0.19 \mu \mathrm{m}, \mathrm{H}$ & $1.2 \mu \mathrm{m}, \mathrm{D}$ & $250 \mathrm{~W}$ \\
\hline $\mathrm{HJ} 13$ & none & $0.13 \mu \mathrm{m}, \mathrm{H}$ & $200 \mathrm{~W}$ \\
\hline
\end{tabular}

Table 1. Summary of deposition parameters of samples used in this study.
Neutron reflectometry[16] has been used for several years in the study of a wide variety of systems[17] principally magnetic materials[18] and polymers.[19] The present measurements were

done on the POSYII instrument at Argonne National Laboratory's Intense Pulsed Neutron Source.[20] A complete explanation of the relationship between the wavelength and angle dependence of the reflected neutrons and the depth profile of a specimen is given in the references[16-19]; only a summary will be provided here.

The index of refraction for neutrons, $n(k)$, can be defined as:

$$
n(k)=\sqrt{1-\frac{4 \pi(b / v)}{k^{2}}}
$$

where $\mathrm{k}$ is the neutron momentum $(2 \pi / \lambda)$ and $b / V$ is the neutron scattering length density given by:

$$
\mathrm{b} / \mathrm{V}=\frac{1}{\mathrm{~V}} \sum_{\substack{\text { nuclear } \\ \text { spedies }}} \mathrm{N}_{i} \mathrm{~b}_{i}
$$

where $\mathrm{b}$ is the neutron scattering length of each nuclear species $i$. The scattering lengths of all nuclear isotopes are on the order $10^{-5} \AA$ and can be either positive or negative (usually positive). Note that for a positive scattering length density the index of refraction is smaller than unity and that for $k<k_{c}(=\sqrt{ } 4 \pi b / V) n(k)$ is imaginary. The scattering lengths for $H, D$ and Si are $-3.74 \times 10^{-5} \AA, 6.7 \times 10^{-5} \AA$ and $4.15 \times 10^{-5} \AA$, respectively.

The reflectanco for a wave from infinite medium 1 to infinite medium 2 is:

$$
r_{12}=\frac{k_{z 1}-k_{z 2}}{k_{z 1}+k_{z 2}}
$$

where:

$$
\mathrm{k}_{\mathrm{z}}=\frac{2 \pi}{\lambda} \sin \theta
$$

$\theta$ is the angle of incidence (reflection) of the wave (measured from the direction parallel to the interface) and $z$ is the direction normal to the interface. The reflectivity $R\left(k_{z}\right)$ is just the modulus squared of the reflectance and will decrease as $\mathrm{k}_{\mathrm{z}}^{-4}$ for large $\mathrm{k}_{\mathrm{z}}$. It is common therefore to remove this trivial dependence and plot $\mathrm{Rk}^{4} \mathrm{vs}$. $\mathrm{k}$ (we will henceforth drop the subscript $\mathrm{z}$ ). In a sample containing many interfaces there will, of course, be multiple reflections and taking these into account leads to $R(k)$ being a nonlinear transform of $n(z, k)$. The reflectivity can then be calculated by a recursive technique such as the scheme of Parrat.[21] There is no inverse transform, due to the lack of phase information in $R$ and the nonlinearity of the iransform; typically the data are fit to a model for the profile $n(z)$. 


\section{Results}

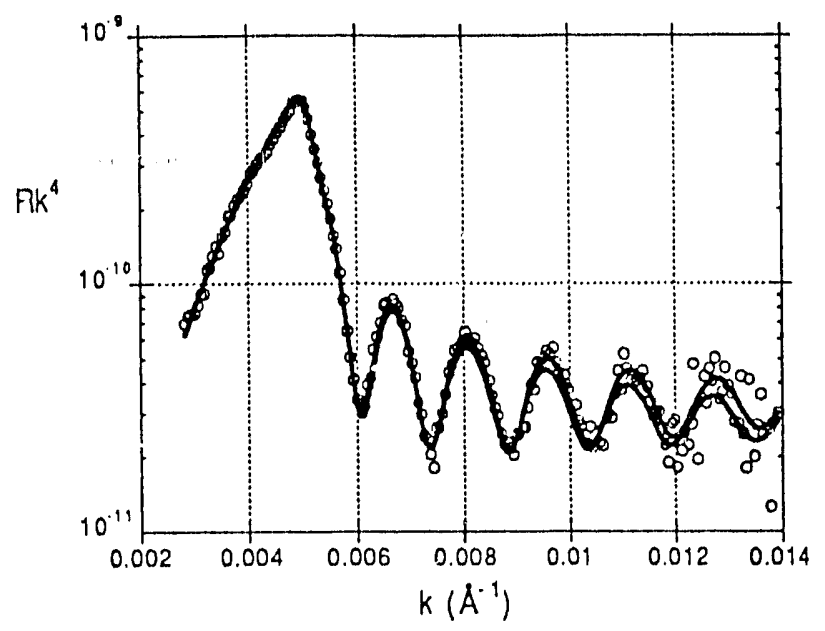

Firgure 1. NR data from sample HJ13. The circles are the experimental data points and the solid lines are least-squares fits as explained in the text.

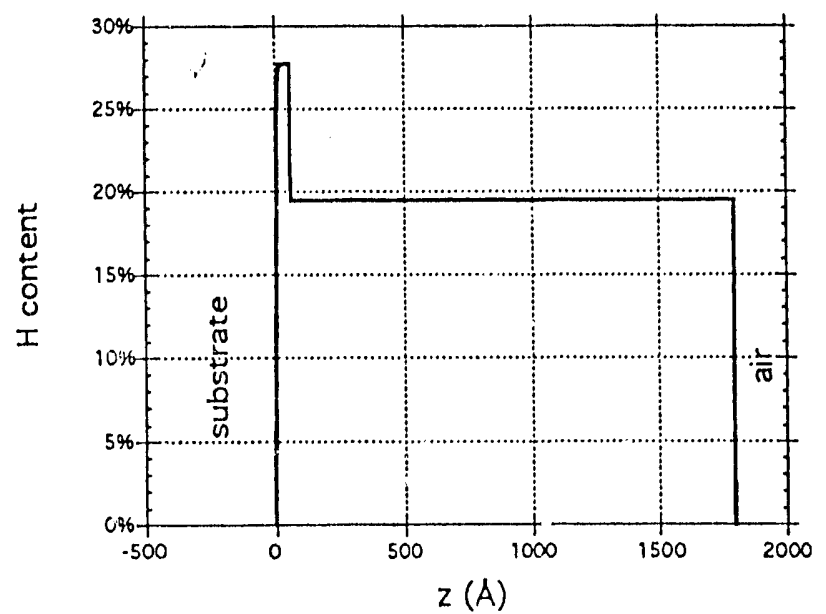

Figure 2. Real-space profile of $\mathrm{H}$ density in sample $\mathrm{HJ} 13, \mathrm{a}-\mathrm{Si}$ density $92 \%$ of $\mathrm{c}$-Si assumed.

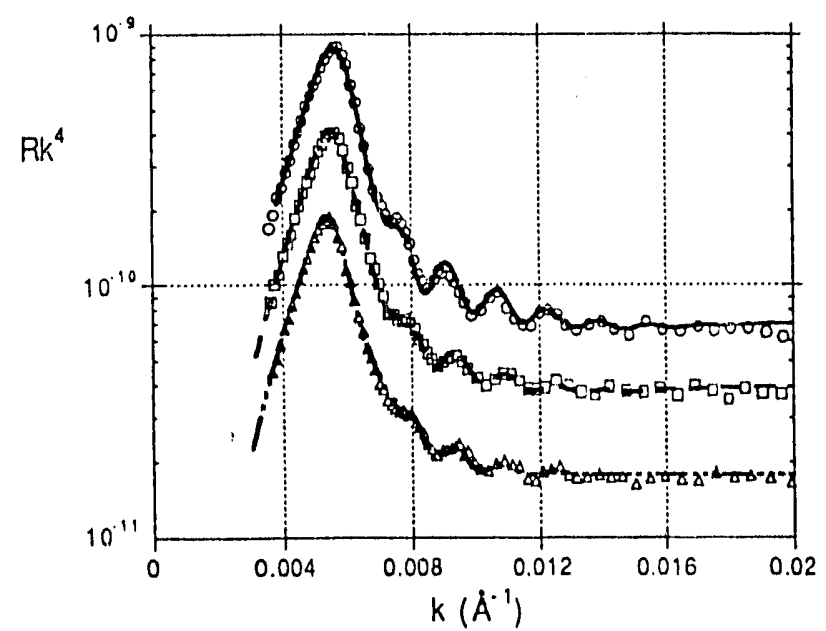

Figure 3. NR data and fits for sample HJ34. Circles:"as prepared," squares: 2 hours @ 270C, uriangles: 4 hours @ 270C.
The neutron reflectivity measured from sample $\mathrm{HJ} 13$ is shown in Figure 1. Oscillations in the data are due to constructive and destructive interference of waves reflected from the air/sample and the a-Si:H/substrate interfaces. Also shown in Figure 1 are fits to the data generated from two models. The first is a single uniform layer deposited on the $c-\mathrm{Si}$ substrate; the second has two such layers. Associated with each layer is a roughness taken as a Gaussian height-height correlation function.

Figure 2 shows the $\mathrm{H}$ density profile associated with the two-layer model. By incorporating a thin layer of higher $\mathrm{H}$ content next to the c-Si substrate, the twolayer model explicitly accounts for the brief over-pressure of $\mathrm{H}$ at the beginning of the deposition procedure. This model fits the data significantly better than the singlelayer model $\left(\chi^{2}=2.7\right.$ vs. 4.3$)$, demonstrating the high resolution and sensitivity of the NR technique. In either case, the total thickness of the a-Si layer is 1760 $\AA, 500 \AA$ thicker than estimated from the deposition parameters. The "overpressure" layer was $60 \AA$ thick. This sample was too thin for a reliable IR determination of its Si-bonded $\mathrm{H}$ content. The IR spectra of thicker films deposited under similar conditions contained $\approx 20$ at. \% Si-bonded $\mathrm{H}$ and their small-angle $x$-ray scattering spectra indicated a columnar morphology and a microvoid volume fraction of $\approx 8 \%$.[22] Taking the density of the a-Si to be $92 \%$ that of $\mathrm{c}-\mathrm{Si}$, the neutron data indicate a total $\mathrm{H}$ concentration of 19 at. \%. This is good agreement, the NR providing an independent confirmation of IR measurements.

Figure 3 shows the reflectivity data measured from sample HJ34 as deposited and after each of two 2 hour anneals at 270 C. The figure also shows fits to the NR data calculated from a model having $4 \mathrm{a}-\mathrm{Si}: \mathrm{H}, \mathrm{D}$ layers on a $\mathrm{c}-\mathrm{Si}$ substrate. Beginning from the 
substrate, the layers are: a thin region containing high $\mathrm{D}$ content due to overpressuring of the chamber as in sample HJ13 above, a thick $(\approx 12000 \AA)$ a-Si:D layer, another thin "overpressure" layer containing a high $\mathrm{H}$ content and finally a layer of a-Si:H $\approx 1900 \AA$ thick. Each of these layers is taken to have a Gaussian roughness (this is equivalent to having a complementary error function density profile).

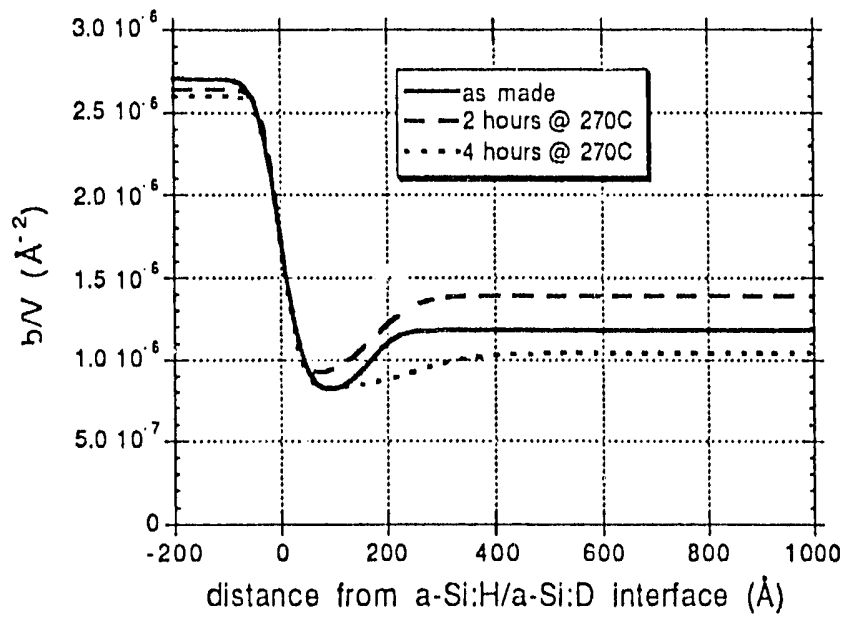

Figure 4a. Scattering length density from fits near a-Si:H/a-Si:D interface.

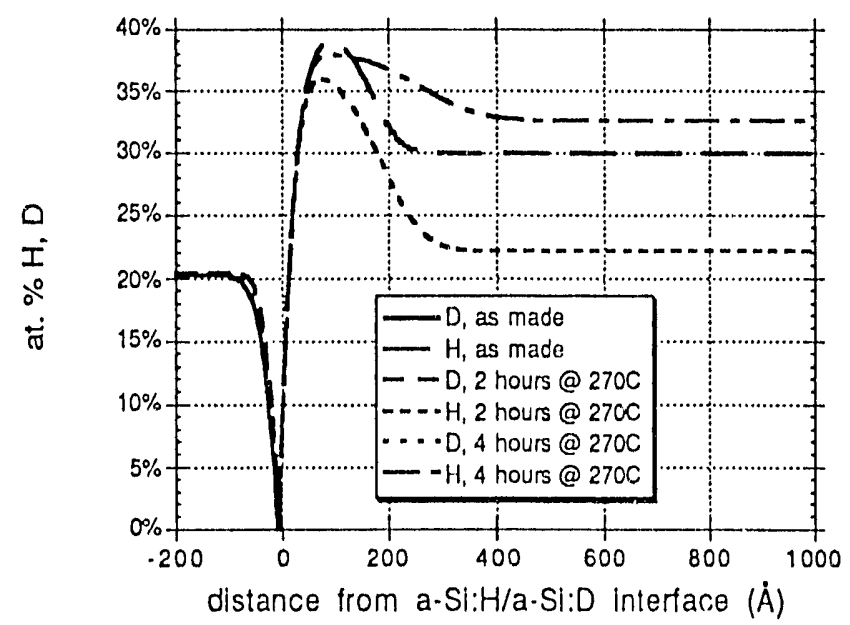

Figure 4b. H, D content near a-Si:H/a-Si:D interface. Note that the D profiles are intentionally scaled to be coincident.

Figure 6 compares the NR from sample HJ34 after a total of 4 and 8 hours at $270 \mathrm{C}$; the data are practically indistinguishable.
Figure 4 a shows the scattering length density profile for the region near the a-Si:D/a-Si:H interface; figure $4 \mathrm{~b}$ shows the resulting $\mathrm{H}$ and $\mathrm{D}$ profiles. In going from the scattering length density profile to the $H / D$ profile, we must assume reasonable values for some of the parameters as the set of variables is underdetermined. We assumed a density of a-Si that is $92 \%$ that of c-Si, or in other words, an $8 \%$ microvoid density. On annealing, the scattering length density in the a-Si:D layer decreased while in the a-Si:H layer it increased. Since upon annealing sinall bubble-like structures (size $\approx$ $1 \mathrm{~mm}$ or less) appeared in the sample and this was accompanied by a marked increase in the smallangle scattering, we assumed that the decrease in scattering length density in the boitom (a-Si:D) layer must be due to a decrease in density of the a-Si matrix. This changes the density from $92 \%$ to $90 \%$ and $88 \%$ of c-Si for the 2 and 4 hours anneals, respectively. There is some scatter in the $\mathrm{H}$ content of the upper layer, but the agreement is acceptable given the nature of this treatment.

Figure 5 shows the interface half-width between the $\mathrm{H}$ overpressure layer and the top (a$\mathrm{Si}: \mathrm{H})$ layer as a function of annealing time. We can estimate a diffusion constant of $8 x$ $10^{-17} \mathrm{~cm}^{2} / \mathrm{sec}$ from this line.

\section{Discussion}

The principal result from this study is that NR has considerable potential for the study of short-range motion in a-Si:H. We are able to determine that, in a sample containing a large microvoid volume fraction, the diffusion process appears to stop sometime between 2 and 4 hours at $270 \mathrm{C}$. This is consistent with a model in which the hydrogen will become trapped in the microvoids after diffusing a short distance.[5] In this sample, this distance was on the order of $100 \AA$. Subsequent annealing at $270 \mathrm{C}$, produced no change in the NR. The estimated diffusion coefficient is quite high for this temperature, but that should also be anticipated for 


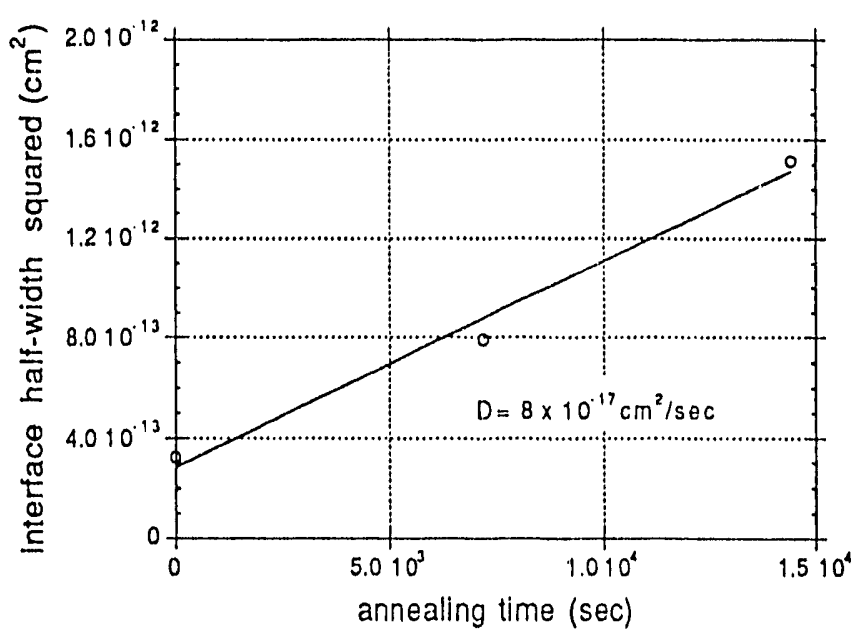

Figure 5. Half-width of interface between "overpressure" a-Si:H layer and top a-Si:H layer as function of annealing time at $270 \mathrm{C}$.

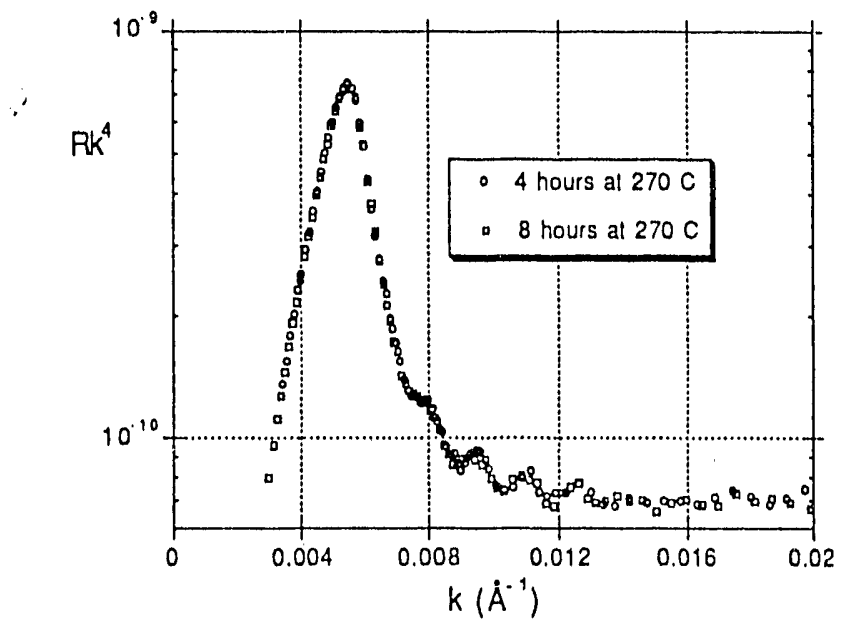

Figure 6. NR from HJ34 showing virtually identical results after 4 and 8 hours at $270 \mathrm{C}$.

Ames was supporte Ames was supported by the US Department of Energy, Basic Energy Sciences, under contracts Nos. W-31-109-ENG-38 and W-7405-ENG-82, respectively. One of us (WDD) also is supported by the NSF under contract No. DMR-8902024.

\section{Ieferences}

1 . D. E. Carlson and C. W. Magee, Appl. Phys. Lett. 33, 81 (1978).

2. W. Beyer, in Tetrahedrally Bonded Amorphous Semiconductors, D. Adler and H. Fritzsche, e(Is. (Plenum, NY), 1985, p. 129 and references therein.

3. R. A. Street, C. C. Tsai, J. Kakalios and W. B. Jackson, Phil. Mag. B 56, 305 (1987).

4. J. Kaklios, R. A. Street, W. B. Jackson and C. C. Tsai, Phys. Rev. Lett. 59, 1037 (1987).

5. I. Shinar, R. Shinar, S. Mitra and J. Y. Kim, Phys. Rev. Lett. 62, 2001 (1989).

6. J. Shinar, R. Shinar, X. L. Wu, S. Mitra and R. F. Girvan, Phys. Rev. B 43, 1631 (1991).

7. X. L. Wu, R. Shinar and J. Shinar, Phys. Rev. B 44, 6161 (1991).

8. X. M. Tang, J. Weber, Y. Baer and F. Finger, Phys. Rev. B 41, 7945 (1990).

9. X. M. Tang, J. Weber, Y. Baer and F. Finger, Phys. Rev. B 42, 7277 (1990).

10. P. V. Santos, N. M. Johnson and R. A. Street, Phys. Rev. Lett. 67, 2686 (1991). 
11. V. Halpern, Phys. Rev. Lett. 67, 611 (1991).

12. W. B. Jackson and C. C. Tsai, to appear in Phys. Rev. B .

13. B. L. Stafford, ed., Stability of Amorphous Silicon Materials and Solar Cells, Amer. Inst. Phys. Conf. Proc. 234 (Amer. Inst. Phys., NY, 1991).

14. M. L. Albers, J. Shinar and H. R. Shanks, J. Appl. Phys. 64, 1859 (1988).

15. M. Cardona, Phys. Status Solidi (b) 118, 463 (1983).

16. G. P. Felcher, R. O. Hilleke, R. K. Crawford, J. Haumann, R. Kleb and G. Ostrowski, Rev. Sci. Instr. 58, 609 (1987).

17. G. P. Felcher and T. P. Russell, eds., Methods of Analysis and Interpretation of Neutron Reflectivity Data, Physica B 173 (1991).

18. G. P. Felcher and Y. Y. Huang, to appear in proceedings of NATO ASI.

19. T. P. Russell, Mat. Sci. Rep. 5, 171 (1990) and references therein.

20. A. Karim, B. H. Arendt, R. Goyette, Y. Y. Huang, R. Kleb and G. P. Felcher, in Methods of Analysis and Interpretation of Neutron Reflectivity Data, G. P. Felcher and T. P. Russell, eds., Physica B 173, 17(1991).

21. L. G. Parrat, Phys. Rev. 95, 359(1954) and L. G. Parrat, J. Chem. Phys. 53, 597(1956).

22. H. Jia, J. Shinar, Y. Chen and D. L. Williamson, this volume. 


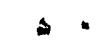
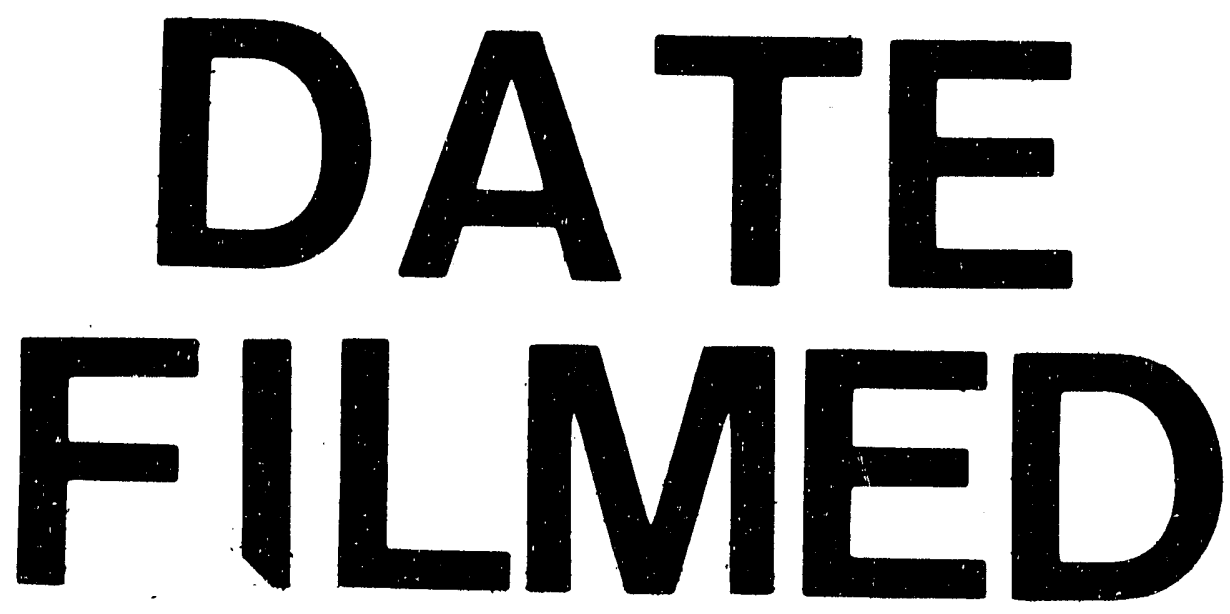

$8 / 17 / 92$

i 
